\title{
Correlates of Cervical Cancer Screening among Vietnamese American Women
}

\author{
Grace X. Ma, ${ }^{1,2}$ Carolyn Y. Fang, ${ }^{3}$ Ziding Feng, ${ }^{4}$ Yin Tan, ${ }^{2}$ Wanzhen Gao, ${ }^{2}$ \\ Shaokui Ge, ${ }^{4}$ and Cuc Nguyen ${ }^{5,6}$ \\ ${ }^{1}$ Department of Public Health, College of Health Professions, Temple University, 1301 Cecil B Moore Avenue, 913 Ritter Annex, \\ Philadelphia, PA 19122, USA \\ ${ }^{2}$ Center for Asian Health, College of Health Professions, Temple University, 1415 N. Broad Street, Suite 116, Philadelphia, \\ PA 19122, USA \\ ${ }^{3}$ Fox Chase Cancer Center, Cancer Prevention and Control Program, 333 Cottman Avenue, Philadelphia, PA 19111, USA \\ ${ }^{4}$ Biostatistics Program, Cancer Prevention Research Program, Fred Hutchinson Cancer Research Center, Department of Biostatistics, \\ University of Washington, P.O. Box 19024, Seattle, WA 98109, USA \\ ${ }^{5}$ Asian Community Health Coalition, 1106 Buttonwood Street, Unit A, Philadelphia, PA 19123, USA \\ ${ }^{6}$ Vietnamese Women Association of Eastern Region, 3 Coventry Circle East, Marlton, NJ 08053, USA
}

Correspondence should be addressed to Grace X. Ma, grace.ma@temple.edu

Received 23 April 2012; Revised 17 July 2012; Accepted 23 July 2012

Academic Editor: Kimlin Ashing-Giwa

Copyright (C) 2012 Grace X. Ma et al. This is an open access article distributed under the Creative Commons Attribution License, which permits unrestricted use, distribution, and reproduction in any medium, provided the original work is properly cited.

\begin{abstract}
Objective. Vietnamese American women are at the greatest risk for cervical cancer but have the lowest cervical cancer screening rates. This study was to determine whether demographic and acculturation, healthcare access, and knowledge and beliefs are associated with a prior history of cervical cancer screening among Vietnamese women. Methods. Vietnamese women $(n=1450)$ from 30 Vietnamese community-based organizations located in Pennsylvania and New Jersey participated in the study and completed baseline assessments. Logistic regression analyses were performed. Results. Overall levels of knowledge about cervical cancer screening and human papillomavirus (HPV) are low. Factors in knowledge, attitude, and beliefs domains were significantly associated with Pap test behavior. In multivariate analyses, physician recommendation for screening and having health insurance were positively associated with prior screening. Conclusion. Understanding the factors that are associated with cervical cancer screening will inform the development of culturally appropriate intervention strategies that would potentially lead to increasing cervical cancer screening rates among Vietnamese women.
\end{abstract}

\section{Introduction}

Asian Americans comprise the fastest growing immigrant population in the United States (USA) and currently number about 18 million or $6 \%$ of the total USA population [1]. Vietnamese Americans represent the fourth largest subgroup of Asian Americans with 1.6 million individuals [2]. A significant proportion of Vietnamese Americans experience serious socioeconomic difficulties, and those residing in the eastern region of the USA report higher levels of poverty and lower levels of educational achievement as compared with other Asian subgroups nationwide or the USA population [3, 4]. Studies have shown that Vietnamese Americans also have higher rates of certain types of cancers [5]. In comparison to other racial and ethnic groups in the USA, Vietnamese women experience the highest incidence rate of invasive cervical cancer $[5,6]$. Due to potential underreporting, the cancer incidence rates among Vietnamese women may be higher [7].

Over the past few decades, significant advances in cervical cancer early detection and prevention have benefitted a large subset of the USA female population by reducing the incidence, prevalence, and mortality rates of this disease; however, these rates have remained elevated in certain population subgroups [8]. These disparities in cervical cancer incidence rates have been attributed, in part, to differences in screening uptake. For example, cervical cancer screening rates are dramatically lower among Vietnamese 
American women compared to women in other ethnic and racial subgroups [9], with a prior study reporting that 1 in 3 Vietnamese women had never had a Papanicolaou (Pap) test [10]. Considering the high rates of cervical cancer among Vietnamese American women [5, 6], cervical cancer screening is a critical public health priority. However, there is a lack of knowledge on how to approach this health issue effectively in this population.

Previous studies have indicated that there are substantial barriers that prevent Vietnamese American women from obtaining Pap tests. In addition to the perceived invasive nature of the Pap test itself, Vietnamese women share with other members of their communities common, often insurmountable barriers, including cultural (modesty), linguistic (limited or no English proficiency), and socioeconomic (poverty) barriers and a pervasive unfamiliarity with the USA healthcare system [11-14]. Although each of these barriers has been found to be associated with screening rates in other populations, the impact of these barriers is more profound on this hard-to-reach ethnic group $[9,15,16]$. Evidence of the profound impact of these barriers is indicated by the fact that a substantial majority of Vietnamese (75\%) residing in the eastern USA are either medically underserved or uninsured and are not participating in mainstream screening and prevention programs [10]. Thus, screening rates in this subgroup are very low [10]. Even though prior studies of cancer screening behaviors have been conducted among Vietnamese Americans residing in the western or southern USA, studies suggest that there are significant geographic variations in cancer screening behaviors and incidence rates $[17,18]$. Hence, there is a need for a better understanding of how these various factors may be associated with cervical cancer screening behaviors of Vietnamese American women residing in the eastern USA, which will inform the development of appropriate strategies that would facilitate the participation of these communities in lifesaving intervention and early detection programs in order to reduce such disparities and improve accessibility and quality of health care for this population.

We have been conducting one of the largest randomized community cervical cancer intervention trials, aimed to increase cervical cancer screening and reduce health system access barriers among medically underserved and lowincome Vietnamese women. This randomized controlled trial (RCT) is a five-year study conducted at 30 Vietnamese community organizations in the eastern region of the USA (PA, NJ). The intervention trial, guided by a conceptual framework derived from the Health Belief Model (HBM) and Social Cognitive Theory (SCT), addresses both individual choices and healthcare system barriers. An overview of the intervention trial and primary results will be reported in a separate publication. The purpose of this paper is to analyze the baseline data in order to: (a) determine whether demographic, health access, and KAB (knowledge, attitudes, and beliefs) factors are associated with prior history of cervical cancer screening among Vietnamese American women and (b) to identify important factors that inform the development of culturally appropriate intervention strategies that would lead to reducing cervical cancer screening disparity among Vietnamese women.

\section{Methods}

2.1. Study Sites and Participants. The Center for Asian Health (Center) has an enduring collaborative relationship with over 250 Asian American organizations represented by the Asian Community Health Coalition (ACHC), a nonprofit 501(c)(3) umbrella organization, established concurrently with the Center in 2000. The Vietnamese community-based organizations (VCOs) included in this study as part of the Coalition serve important social functions, and they represent the ideal milieu for obtaining information on accessibility to needed health services. Their unique status in these communities underscores their importance as an ideal avenue for recruiting community-based participants for the study and intervention delivery. In the present study, participant recruitment took place at 30 Vietnamese CBOs. The CBOs varied in size, with the number of female Vietnamese members in each of these 30 organizations ranging from between 80 to 2500 women. The average age of study participants was 52 years (range: 20 to 70 years). About 42 Vietnamese community leaders were involved in the study. Specifically, they were directly involved in the planning, development, and implementation of the project.

Vietnamese women $(n=1949)$ were recruited from participating community-based Vietnamese community organizations $(n=30)$ and assessed for study eligibility. Of the total recruited and assessed, 1518 met the inclusion criteria of self-identified Vietnamese identity, ages 18 to 70, had not had a Pap-test over the past 12 months, and had not been diagnosed with cervical cancer. Of the total eligible women, 1450 consented, completed the baseline survey, and are included in the data analysis for this paper. Participants were compensated $\$ 30$ for participating in this study. The study was approved by the Temple University Institutional Review Board.

2.2. Data Collection Procedures. Prior to project implementation, community leaders and volunteers participated in training sessions focused on the revisitation of project aims and significance to Vietnamese women, recruitment strategies, and guidelines for administration of the research instrument as well as data collection, accuracy, and confidentiality. All measures in English were translated, back-translated, and pretested in Vietnamese to ensure the scientific and cultural appropriateness of the instrument for community Vietnamese participants. The 20-30-minute baseline survey was provided in Vietnamese and English versions, and bilingual assistance was available at all sites.

2.3. Measures. The measures collected at baseline assessment include (1) demographics and acculturation; (2) health care access; (3) health behavior and Pap test history; (4) perceptions related to health belief model constructs; (5) knowledge, attitudes, and beliefs of Vietnamese women about cervical cancer; (6) human papillomavirus (HPV-) related 
TABle 1: Associations between demographic characteristics and Papanicolaou (PAP) testing among Vietnamese women.

\begin{tabular}{|c|c|c|c|c|c|c|c|c|}
\hline \multirow{3}{*}{ Demographic factors } & \multicolumn{4}{|c|}{ Ever had a Pap test } & \multicolumn{4}{|c|}{ Odds Ratios } \\
\hline & \multicolumn{2}{|c|}{ Yes } & \multicolumn{2}{|c|}{ No } & \multicolumn{2}{|c|}{ Single factors } & \multicolumn{2}{|c|}{ Multiple factors by forward selection } \\
\hline & No. & $\%$ & No. & $\%$ & Point estimate & $95 \% \mathrm{CI}^{\mathrm{b}}$ & Point estimate & $95 \% \mathrm{CI}^{\mathrm{b}}$ \\
\hline Age: & 760 & 52.85 & 678 & 47.15 & $* * *^{\mathrm{a}}$ & & $* * *$ & \\
\hline$>60$ years old & 243 & 61.36 & 153 & 38.64 & 1.00 & Reference & 1.00 & Reference \\
\hline $51-60$ years old & 218 & 56.92 & 165 & 43.08 & 0.83 & $0.63 \sim 1.11$ & $0.63^{* *}$ & $0.45 \sim 0.89$ \\
\hline $41-50$ years old & 166 & 53.90 & 142 & 46.10 & $0.74^{* *}$ & $0.54 \sim 1.00$ & $0.51^{* * *}$ & $0.35 \sim 0.74$ \\
\hline $18-40$ years old & 133 & 37.89 & 218 & 62.11 & $0.38^{* * *}$ & $0.29 \sim 0.52$ & $0.26^{* * *}$ & $0.17 \sim 0.38$ \\
\hline Education level & 738 & 53.40 & 644 & 46.60 & $* * *$ & & $* * *$ & \\
\hline Below high school & 211 & 47.50 & 251 & 52.50 & 1.00 & Reference & 1.00 & Reference \\
\hline High school & 472 & 57.42 & 350 & 42.58 & $1.60^{* * *}$ & $1.28 \sim 2.02$ & $1.62^{* * *}$ & $1.23 \sim 2.14$ \\
\hline Higher education & 55 & 56.12 & 43 & 43.88 & 1.52 & $0.98 \sim 2.36$ & $2.44^{* * *}$ & $1.35 \sim 4.44$ \\
\hline Employment status & 766 & 53.27 & 672 & 46.73 & $* * *$ & & $* *$ & \\
\hline Employed & 493 & 56.15 & 385 & 43.85 & 1.00 & Reference & 1.00 & Reference \\
\hline Unemployed & 49 & 33.79 & 96 & 66.21 & $0.40^{* * *}$ & $0.28 \sim 0.58$ & $0.51^{* *}$ & $0.34 \sim 0.78$ \\
\hline Retired & 48 & 66.67 & 24 & 33.33 & 1.56 & $0.94 \sim 2.60$ & 1.34 & $0.71 \sim 2.53$ \\
\hline Homemaker & 176 & 51.31 & 167 & 48.69 & 0.82 & $0.64 \sim 1.06$ & 0.76 & $0.56 \sim 1.05$ \\
\hline English speaking ability & 766 & 53.05 & 678 & 46.95 & $* *$ & & $* * *$ & \\
\hline Not at all & 269 & 48.47 & 286 & 51.51 & 1.00 & Reference & 1.00 & Reference \\
\hline Not well & 451 & 56.73 & 344 & 43.27 & $1.39^{* *}$ & $1.12 \sim 1.73$ & $1.85^{* * *}$ & $1.39 \sim 2.47$ \\
\hline Well & 46 & 48.94 & 48 & 51.06 & 1.02 & $0.66 \sim 1.58$ & 1.77 & $0.97 \sim 3.24$ \\
\hline Current marital states & 765 & 53.13 & 675 & 46.88 & $* * *$ & & $* * *$ & \\
\hline Married/living as married & 606 & 55.85 & 479 & 44.15 & 1.00 & Reference & 1.00 & Reference \\
\hline Never married & 71 & 37.57 & 118 & 62.43 & $0.48^{* * *}$ & $0.35 \sim 0.65$ & $0.53^{* *}$ & $0.36 \sim 0.78$ \\
\hline Divorced/separated & 25 & 39.68 & 38 & 60.32 & $0.52 *$ & $0.31 \sim 0.87$ & $0.45^{* *}$ & $0.25 \sim 0.82$ \\
\hline Widowed & 63 & 61.76 & 39 & 38.24 & 1.28 & $0.84 \sim 1.94$ & 1.30 & $0.81 \sim 2.10$ \\
\hline Born in USA & 762 & 53.03 & 675 & 46.97 & $* * *$ & & $* *$ & \\
\hline No & 705 & 52.14 & 647 & 47.86 & 1.00 & Reference & 1.00 & Reference \\
\hline Yes & 57 & 67.06 & 28 & 32.94 & $1.87^{* *}$ & $1.17 \sim 2.97$ & $2.22 * *$ & $1.34 \sim 3.66$ \\
\hline Years lived in USA & 769 & 53.03 & 681 & 46.97 & $* * *$ & & & \\
\hline $1 \sim 10$ years & 226 & 44.58 & 281 & 55.42 & 1.00 & Reference & & \\
\hline $11 \sim 20$ years & 476 & 56.13 & 372 & 43.87 & $1.59^{* * *}$ & $1.28 \sim 1.99$ & & \\
\hline $21 \sim 35$ years & 67 & 70.53 & 28 & 29.47 & $2.97^{* * *}$ & $1.85 \sim 4.78$ & & \\
\hline English reading ability & 762 & 52.88 & 679 & 47.12 & $*$ & & & \\
\hline Not at all & 403 & 49.94 & 404 & 50.06 & 1.00 & Reference & & \\
\hline Not well & 322 & 57.30 & 240 & 42.70 & $1.35^{* *}$ & $1.08 \sim 1.67$ & & \\
\hline Well & 37 & 51.39 & 35 & 48.61 & 1.06 & $0.65 \sim 1.72$ & & \\
\hline
\end{tabular}

Note: ${ }^{a} * * *, * *, *$ indicating significance levels of $\leq 0.001, \leq 0.01$, and $\leq 0.05$, respectively;

It was the overall significance when following the factor category names; otherwise, it was the significance of the factor level.

${ }^{\mathrm{b}} \mathrm{CI}=$ confidence interval.

questions. Our research team investigators hypothesized that these factors mentioned above would affect cervical cancer screening behaviors by serving as either barriers or facilitators to health-seeking behavior change. This paper primarily examines the association of demographic and acculturation characteristics (measured using 8 items), healthcare access barriers (6 items), and knowledge, attitudes, and beliefs about cervical cancer screening (13 items) and HPV-specific knowledge (10 items) with ever having had a Pap test. These measures were validated in a number of our previous studies [10,13, 19-21]. The findings of HBM constructs and other variables' association with cervical cancer screening behaviors are being reported in a separate publication [22].

2.4. Statistical Analyses Plan. Univariate logistic regression was used to examine the association between the probability of ever having had a Pap smear test and each variable in the domains of demographic variables, access barriers, knowledge, attitude, and beliefs about cervical cancer. The strength of association was expressed as odds ratio and its 95\% confidence interval. Both unadjusted odds ratio and odds ratio adjusted for demographic variables were reported. To examine whether variables in a domain contributed 
TABLe 2: Associations between Papanicolaou (PAP) testing and access barriers.

\begin{tabular}{|c|c|c|c|c|c|c|c|c|}
\hline \multirow{3}{*}{ Access factors } & \multicolumn{4}{|c|}{ Ever had a Pap test } & \multicolumn{2}{|c|}{ Unadjusted odds ratios } & \multicolumn{2}{|c|}{ Adjusted odds ratios } \\
\hline & \multicolumn{2}{|c|}{ Yes } & \multicolumn{2}{|c|}{ No } & \multirow{2}{*}{ Point estimate } & \multirow{2}{*}{$95 \% \mathrm{CI}$} & \multirow{2}{*}{ Point estimate } & \multirow{2}{*}{$95 \% \mathrm{CI}$} \\
\hline & No. & $\%$ & No. & $\%$ & & & & \\
\hline Current health insurance & 767 & 53.26 & 673 & 46.74 & $* * *^{\mathrm{a}, \mathrm{b}}$ & & $* * *$ & \\
\hline No & 224 & 35.22 & 412 & 64.78 & 1.00 & Reference & 1.00 & Reference \\
\hline Yes & 543 & 67.54 & 261 & 32.46 & $3.83^{* * *}$ & $3.07 \sim 4.77$ & $3.41^{* * *}$ & $2.64 \sim 4.39$ \\
\hline Have a healthcare provider & 753 & 53.44 & 656 & 46.56 & $* * *$ & & $* * *$ & \\
\hline No & 105 & 31.82 & 225 & 68.18 & 1.00 & Reference & 1.00 & Reference \\
\hline Yes & 648 & 60.06 & 431 & 39.94 & 3.22 & $2.48 \sim 4.19^{* * *}$ & $2.27 * * *$ & $1.67 \sim 3.08$ \\
\hline Doctor recommendation for testing & 734 & 52.92 & 653 & 47.08 & $* * *$ & & $* * *$ & \\
\hline No & 550 & 46.37 & 627 & 53.27 & 1.00 & Reference & 1.00 & Reference \\
\hline Yes & 184 & 87.62 & 26 & 12.38 & $8.07^{* * *}$ & $5.27 \sim 12.36$ & $9.00 * * *$ & $5.60 \sim 14.44$ \\
\hline Doctor's spoken language & 711 & 57.29 & 530 & 42.71 & $* * *$ & & & \\
\hline Vietnamese & 496 & 53.97 & 423 & 46.03 & 1.00 & Reference & 1.00 & Reference \\
\hline English and Vietnamese & 34 & 57.63 & 25 & 42.37 & $1.16^{* *}$ & $0.68 \sim 1.98$ & 1.64 & $0.89 \sim 3.02$ \\
\hline English & 181 & 68.82 & 82 & 31.18 & $1.88^{* * *}$ & $1.41 \sim 2.52$ & $2.10^{* * *}$ & $1.47 \sim 2.99$ \\
\hline Difficulty getting time off to see doctor & 751 & 53.38 & 656 & 46.62 & $* * *$ & & $* * *$ & \\
\hline No & 558 & 56.65 & 427 & 43.35 & 1.00 & Reference & 1.00 & Reference \\
\hline Yes & 193 & 45.73 & 229 & 54.27 & $0.65^{* * *}$ & $0.51 \sim 0.81$ & $0.70^{* *}$ & $0.54 \sim 0.89$ \\
\hline Preference for doctor's gender for a vaginal exam & 761 & 53.74 & 655 & 46.26 & $* * *$ & & $* * *$ & \\
\hline Female & 418 & 54.93 & 431 & 50.77 & 1.00 & Reference & 1.00 & Reference \\
\hline Male & 33 & 66.00 & 17 & 34.00 & $2.00^{*}$ & $1.10 \sim 3.65$ & $2.41^{*}$ & $1.20 \sim 4.85$ \\
\hline Does not matter & 310 & 59.96 & 207 & 40.04 & $1.54^{* * *}$ & $1.24 \sim 1.93$ & $1.62^{* * *}$ & $1.26 \sim 2.08$ \\
\hline
\end{tabular}

Note: ${ }^{\mathrm{a}} * *, * *, *$ indicating significance levels of $\leq 0.001, \leq 0.01$, and $\leq 0.05$, respectively.

${ }^{b}$ It was the overall significance when following the factor category names; otherwise, it was the significance of the factor level.

independently to Pap test behavior, a forward selection logistic regression model was used. All demographic variables significant in the univariate logistic regression model were included in the multivariate model. All data analyses were performed using SAS 9.2 (SAS Institute Inc. Cary, NC, USA).

\section{Results}

At baseline, 53.03\% (769/1450) reported ever having had a Pap test. Among demographic variables (Table 1), Vietnamese women in the 18-40 age group, who did not speak English at all, were unemployed, never married or divorced/separated, had below high school education, and lived in the USA 10 years or less, were less likely to have had a Pap smear test $(P<0.01)$ as compared to their peers. Women who were born in the USA, lived in the USA for more than 20 years and had some English reading ability, had a greater likelihood of having ever received a Pap smear test $(P<0.01)$. In multivariate analyses, younger age, low education, unemployment, being married, English speaking ability, and country of birth remained significantly associated with prior Pap test behavior.

With regard to access barriers (Table 2), Vietnamese women who did not have insurance, did not visit a physician regularly or could not get time off for a doctor's appointment were less likely to have ever had a Pap test $(P<0.001)$, while women, who had received a doctor recommendation for a Pap test, who visited doctors speaking English or both
English and Vietnamese or who did not have a preference regarding the gender of their doctors, were likely to have had a Pap test in the past $(P<0.001)$. Specifically, receiving a doctor's recommendation for a Pap test was the strongest predictor of obtaining a Pap test (adjusted OR $=9.00,95 \%$ $\mathrm{CI}=5.60-14.44, P<0.001$ ), with $87.6 \%$ of those who received a recommendation reporting that they had had a Pap test compared to $46.4 \%$ of those who had not received a doctor's recommendation for testing. Insurance was the second strongest factor (adjusted $\mathrm{OR}=3.41,95 \% \mathrm{CI}=2.64$ $4.39, P<0.001)$, nearly doubling the Pap test rate $(67.5 \%$ versus $35.2 \%$ ).

All 11 items in the knowledge, attitude, and beliefs domain were significantly associated with Pap test behavior $(P<0.001)$ (Table 3). In particular, it should be noted that the majority of women $(65.8 \%)$ held the belief that you only see doctors when you're sick, whereas relatively few women believed that women older than 21 should be regularly screened $(38.2 \%)$ and that screening was necessary for women not engaging in sexual activities (26.1\%). Further, knowledge of cervical cancer symptoms was low in this group, and only $22.7 \%$ had ever heard of HPV. In univariate analyses, women with a belief that cancer can be cured if detected early, belief that a Pap test can detect cancer early and prolong time, belief that women over age 21 should have a Pap test regularly, or belief that cancer incidence increases with age, were about twice as likely to have had a Pap test compared to women who did not hold these beliefs. 
TABLe 3: Associations between Papanicolaou (PAP) testing and knowledge, attitudes, and beliefs.

\begin{tabular}{|c|c|c|c|c|c|c|c|c|c|}
\hline \multirow{3}{*}{ Beliefs } & \multirow{3}{*}{$\begin{array}{c}\text { Total } \\
\%\end{array}$} & \multicolumn{4}{|c|}{ Ever had a Pap test } & \multicolumn{2}{|c|}{ Unadjusted odds ratios } & \multicolumn{2}{|c|}{ Adjusted odds ratios } \\
\hline & & \multicolumn{2}{|c|}{ Yes } & \multicolumn{2}{|c|}{ No } & \multirow{2}{*}{ Point estimate } & \multirow{2}{*}{$95 \% \mathrm{CI}$} & \multirow{2}{*}{ Point estimate } & \multirow{2}{*}{$95 \%$ CI } \\
\hline & & No. & $\%$ & No. & $\%$ & & & & \\
\hline God or higher power controls cancer & & 764 & 53.58 & 662 & 46.42 & $* * *^{\mathrm{a}, \mathrm{b}}$ & & $* * *$ & \\
\hline Yes & 21.0 & 148 & 49.50 & 151 & 50.50 & 1.00 & Reference & 1.00 & Reference \\
\hline Do not know & 41.1 & 262 & 44.63 & 325 & 55.37 & 0.82 & $0.62 \sim 1.09$ & 0.81 & $0.59 \sim 1.09$ \\
\hline No & 37.8 & 354 & 65.56 & 186 & 34.44 & $1.94^{* * *}$ & $1.46 \sim 2.59$ & $2.06^{* * *}$ & $1.49 \sim 2.84$ \\
\hline Can have cancer but no symptoms & & 762 & 53.51 & 662 & 46.49 & $* * *$ & & $* * *$ & \\
\hline Yes & 36.6 & 343 & 65.71 & 179 & 34.29 & 1.00 & Reference & 1.00 & Reference \\
\hline Do not know & 46.1 & 270 & 41.10 & 387 & 58.90 & $0.36^{* * *}$ & $0.29 \sim 0.46$ & $0.36^{* * *}$ & $0.27 \sim 0.47$ \\
\hline No & 17.2 & 149 & 60.82 & 96 & 39.18 & 0.81 & $0.59 \sim 1.11$ & 0.86 & $0.60 \sim 1.21$ \\
\hline See doctor only when sick & & 762 & 53.51 & 662 & 46.49 & $* * *$ & & $* * *$ & \\
\hline Yes & 65.8 & 459 & 48.93 & 479 & 51.07 & 1 & Reference & 1.00 & Reference \\
\hline Do not know & 10.8 & 68 & 44.16 & 86 & 55.84 & 0.83 & $0.59 \sim 1.16$ & 0.94 & $0.64 \sim 1.39$ \\
\hline No & 23.3 & 235 & 70.78 & 97 & 29.22 & $2.53^{* * *}$ & $1.93 \sim 3.31$ & $3.03^{* * *}$ & $2.21 \sim 4.18$ \\
\hline Cancer can be cured if detected early & & 765 & 53.61 & 662 & 46.39 & $* * *$ & & $* * *$ & \\
\hline Yes & 49.0 & 478 & 68.48 & 220 & 31.52 & 1.00 & Reference & 1.00 & Reference \\
\hline Do not know & 47.3 & 268 & 39.64 & 408 & 60.36 & $0.30 * * *$ & $0.24 \sim 0.38$ & $0.31^{* * *}$ & $0.24 \sim 0.40$ \\
\hline No & 3.7 & 19 & 35.85 & 34 & 64.15 & $0.26^{* * *}$ & $0.14 \sim 0.46$ & $0.27^{* * *}$ & $0.14 \sim 0.52$ \\
\hline $\begin{array}{l}\text { Women } 21 \text { or older should have Pap test } \\
\text { regularly }\end{array}$ & & 765 & 53.87 & 655 & 46.13 & $* * *$ & & $* * *$ & \\
\hline Yes & 38.2 & 389 & 71.77 & 153 & 28.23 & 1.00 & Reference & 1.00 & Reference \\
\hline Do not know & 54.8 & 337 & 43.26 & 442 & 56.74 & $0.30^{* * *}$ & $0.24 \sim 0.38$ & 0.29 & $0.22 \sim 0.38$ \\
\hline No & 7.0 & 39 & 39.39 & 60 & 60.61 & $0.26^{* * *}$ & $0.16 \sim 0.40$ & $0.27^{* * *}$ & $0.17 \sim 0.45$ \\
\hline $\begin{array}{l}\text { Pap test necessary for women not } \\
\text { engaging in sexual activities }\end{array}$ & & 764 & 53.65 & 660 & 46.35 & $* * *$ & & $* * *$ & \\
\hline Yes & 26.1 & 265 & 71.24 & 107 & 28.76 & 1.00 & Reference & 1.00 & Reference \\
\hline Do not know & 64.0 & 428 & 46.93 & 484 & 53.07 & $0.36^{* * *}$ & $0.28 \sim 0.46$ & $0.32^{* * *}$ & $0.23 \sim 0.43$ \\
\hline No & 9.8 & 71 & 50.71 & 69 & 49.29 & $0.42^{* * *}$ & $0.28 \sim 0.62$ & $0.43^{* * *}$ & $0.27 \sim 0.67$ \\
\hline $\begin{array}{l}\text { Women under } 21 \text { but sexually active for } 3 \\
\text { years need Pap test }\end{array}$ & & 764 & 53.69 & 659 & 46.31 & $* * *$ & & $* * *$ & \\
\hline Yes & 26.2 & 264 & 70.78 & 109 & 52.70 & 1.00 & Reference & 1.00 & Reference \\
\hline Do not know & 62.5 & 421 & 47.30 & 469 & 29.22 & $0.37^{* * *}$ & $0.29 \sim 0.48$ & $0.32^{* * *}$ & $0.24 \sim 0.44$ \\
\hline No & 11.2 & 79 & 49.38 & 81 & 50.63 & $0.40^{* * *}$ & $0.28 \sim 0.59$ & $0.41^{* * *}$ & $0.27 \sim 0.63$ \\
\hline $\begin{array}{l}\text { Pap test is necessary for women who no } \\
\text { longer menstruate }\end{array}$ & & 758 & 53.49 & 659 & 46.51 & $* * *$ & & $* * *$ & \\
\hline Yes & 68.1 & 553 & 57.31 & 412 & 42.69 & 1.00 & Reference & 1.00 & Reference \\
\hline Do not know & 27.0 & 174 & 45.55 & 208 & 54.45 & $0.62^{* * *}$ & $0.49 \sim 0.79$ & $0.54^{* * *}$ & $0.41 \sim 0.70$ \\
\hline No & 5.0 & 31 & 44.29 & 39 & 55.71 & $0.59^{*}$ & $0.36 \sim 0.97$ & 0.68 & $0.40 \sim 1.18$ \\
\hline Cancer affects women of all ages & & 762 & 53.59 & 660 & 46.41 & $* * *$ & & $* * *$ & \\
\hline Yes & 45.6 & 443 & 68.26 & 206 & 31.74 & 1.00 & Reference & 1.00 & Reference \\
\hline Do not know & 46.0 & 274 & 41.96 & 379 & 58.04 & $0.34^{* * *}$ & $0.27 \sim 0.42$ & $0.37^{* * *}$ & $0.28 \sim 0.47$ \\
\hline No & 8.4 & 45 & 37.50 & 75 & 62.50 & $0.28^{* * *}$ & $0.19 \sim 0.42$ & $0.28^{* * *}$ & $0.18 \sim 0.44$ \\
\hline $\begin{array}{l}\text { Pap test can detect cancer early and } \\
\text { prolong life }\end{array}$ & & 762 & 53.66 & 658 & 46.34 & $* * *$ & & $* * *$ & \\
\hline Yes & 45.0 & 453 & 70.89 & 186 & 29.11 & 1.00 & Reference & 1.00 & Reference \\
\hline Do not know & 49.4 & 288 & 41.03 & 414 & 58.97 & $0.29^{* * *}$ & $0.23 \sim 0.36$ & $0.30^{* * *}$ & $0.23 \sim 0.38$ \\
\hline No & 5.5 & 21 & 26.58 & 58 & 73.42 & $0.15^{* * *}$ & $0.09 \sim 0.25$ & $016^{* * *}$ & $0.09 \sim 0.29$ \\
\hline
\end{tabular}


TABle 3: Continued.

\begin{tabular}{|c|c|c|c|c|c|c|c|c|c|}
\hline \multirow{3}{*}{ Beliefs } & \multirow{3}{*}{$\begin{array}{c}\text { Total } \\
\%\end{array}$} & \multicolumn{4}{|c|}{ Ever had a Pap test } & \multicolumn{2}{|c|}{ Unadjusted odds ratios } & \multicolumn{2}{|c|}{ Adjusted odds ratios } \\
\hline & & \multicolumn{2}{|c|}{ Yes } & \multicolumn{2}{|c|}{ No } & \multirow{2}{*}{ Point estimate } & \multirow{2}{*}{$95 \% \mathrm{CI}$} & \multirow{2}{*}{ Point estimate } & \multirow{2}{*}{$95 \% \mathrm{CI}$} \\
\hline & & No. & $\%$ & No. & $\%$ & & & & \\
\hline Cancer risk increases with increasing age & & 759 & 53.45 & 661 & 46.55 & $* * *$ & & $* * *$ & \\
\hline Yes & 32.3 & 328 & 71.30 & 132 & 28.70 & 1.00 & Reference & 1.00 & Reference \\
\hline Do not know & 59.6 & 390 & 46.04 & 457 & 53.96 & $0.34^{* * *}$ & $0.27 \sim 0.44$ & $0.33^{* * *}$ & $0.25 \sim 0.44$ \\
\hline No & 8.0 & 41 & 36.28 & 72 & 63.72 & $0.23^{* * *}$ & $0.15 \sim 0.35$ & $0.23^{* * *}$ & $0.15 \sim 0.37$ \\
\hline \multicolumn{10}{|l|}{ Four symptoms of cervical cancer } \\
\hline No. 1 Vaginal bleeding & & 769 & 53.03 & 681 & 46.97 & $* * *$ & & $* * *$ & \\
\hline No & 78.6 & 556 & 48.73 & 585 & 51.27 & 1.00 & Reference & 1.00 & Reference \\
\hline Yes & 21.3 & 213 & 68.93 & 96 & 31.07 & $2.33^{* * *}$ & $1.79 \sim 3.05$ & $1.88^{* * *}$ & $1.39 \sim 2.53$ \\
\hline No. 2 Unusual vaginal discharge & & 769 & 53.03 & 681 & 46.97 & $* * *$ & & $* * *$ & \\
\hline No & 74.8 & 531 & 48.94 & 554 & 51.06 & 1.00 & Reference & 1.00 & Reference \\
\hline Yes & 25.1 & 238 & 65.21 & 127 & 34.79 & $1.96^{* * *}$ & $1.53 \sim 2.50$ & $1.70^{* * *}$ & $1.29 \sim 2.26$ \\
\hline No. 3 Pelvic pain & & 769 & 53.03 & 681 & 46.97 & $* * *$ & & * & \\
\hline No & 86.4 & 643 & 51.32 & 610 & 48.68 & 1.00 & Reference & 1.00 & Reference \\
\hline Yes & 13.5 & 126 & 63.96 & 71 & 36.04 & $1.68^{* * *}$ & $1.23 \sim 2.30$ & $1.59^{*}$ & $1.12 \sim 2.27$ \\
\hline No. 4 Pain during sexual intercourse & & 769 & 53.03 & 681 & 46.97 & * & & $*$ & \\
\hline No & 8.51 & 638 & 51.70 & 596 & 48.30 & 1.00 & Reference & 1.00 & Reference \\
\hline Yes & 14.8 & 131 & 60.65 & 85 & 39.35 & $1.44^{*}$ & $1.07 \sim 1.93$ & 1.34 & $0.96 \sim 1.88$ \\
\hline Ever heard of HPV & & 744 & 53.30 & 652 & 46.70 & $* * *$ & & $* * *$ & \\
\hline No & 77.2 & 502 & 46.57 & 576 & 53.43 & 1.00 & Reference & 1.00 & Reference \\
\hline Yes & 22.7 & 242 & 76.10 & 76 & 23.90 & $3.65^{* * *}$ & $2.75 \sim 4.85$ & $4.49^{* * *}$ & $3.15 \sim 6.38$ \\
\hline
\end{tabular}

Note: ${ }^{a} * * *, * *, *$ indicating significance levels of $\leq 0.001, \leq 0.01$, and $\leq 0.05$, respectively.

${ }^{b}$ It was the overall significance when following the factor category names; otherwise, it was the significance of the factor level.

With respect to HPV-specific knowledge (Table 4), women who knew that HPV causes cervical cancer and is sexually transmitted were more likely to have had a Pap test compared to women who did not know this information. Similarly, women who were familiar with HPV risk factors (such as having multiple sexual partners) were more likely to have obtained screening compared with women who did not know the risk factors. Finally, women who believed that HPV infection is rare were less likely to have been previously screened.

The findings from multivariate analyses are presented in Table 5. Given that there was a considerable number of women who did not respond to any of the HPV knowledge items (Table 4), we did not include those items in the multivariate analyses. In multivariate analyses, receiving a doctor's recommendation for the Pap test, having insurance, and having time-off to see doctors remained strongly associated with having had a prior Pap test $(P<0.001)$, suggesting that they are independently associated with Pap test behavior even after adjusting for the other access barriers and for demographic variables. In addition, six variables from the knowledge, attitudes, and beliefs domain remained highly significant $(P<0.001$, Table 5$)$ in the multivariate logistic model, indicating their independent contributions to Pap test behavior.

\section{Discussion}

One of the most critical issues in cervical cancer prevention among Vietnamese American women over the past two decades is the persistently low Pap screening rate despite significantly high incidence rate of cervical cancer. Specific challenges and barriers to obtaining screening need to be examined and addressed in this underserved population. Although previous studies in the western region of the USA laid the groundwork for interventions [23-26], they rarely addressed both individual and system barriers that are associated with cervical cancer screening behaviors among Vietnamese women in a single study with a large sample size $(n=$ 1450). In addition, geographic differences may be important to consider, as geographic variations may exist regarding access to healthcare providers and/or healthcare staff who can provide bilingual services. Gresenz et al. (2009) have demonstrated that the considerable language obstacles that exist within some immigrant populations can serve as critical barriers to healthcare and uptake of screening tests [27].

Findings from this population suggest that younger Vietnamese women are less likely to have ever had a Pap smear compared to their older ( $>60$ years) counterparts. This finding is in contrast to a number of studies [16, 2830 ], which have been summarized in several recent reviews 
TABle 4: Associations between Papanicolaou (PAP) testing and HPV-specific knowledge.

\begin{tabular}{|c|c|c|c|c|c|c|c|c|}
\hline \multirow{3}{*}{ Associated factors } & \multicolumn{4}{|c|}{ Ever had a Pap test } & \multicolumn{2}{|c|}{ Unadjusted odds ratios } & \multicolumn{2}{|c|}{ Adjusted odds ratios } \\
\hline & \multicolumn{2}{|c|}{ Yes } & \multicolumn{2}{|c|}{ No } & \multirow{2}{*}{ Point estimate } & \multirow{2}{*}{$95 \%$ CI } & \multirow{2}{*}{ Point estimate } & \multirow{2}{*}{$95 \%$ CI } \\
\hline & No. & $\%$ & No. & $\%$ & & & & \\
\hline HPV causes cervical cancer & 702 & 54.67 & 582 & 45.33 & & & & \\
\hline No & 446 & 47.35 & 496 & 52.65 & 1.00 & Reference & 1.00 & Reference \\
\hline Yes & 256 & 74.85 & 86 & 25.15 & 3.31 & $2.51 \sim 4.36^{* * *}$ & 3.76 & $2.70 \sim 5.24^{* * *}$ \\
\hline HPV is sexually transmitted & 688 & 54.43 & 576 & 45.57 & & & & \\
\hline No & 414 & 46.46 & 477 & 53.54 & 1.00 & Reference & 1.00 & Reference \\
\hline Yes & 274 & 73.46 & 99 & 26.54 & 3.19 & $2.45 \sim 4.16^{* * *}$ & 3.86 & $2.78 \sim 5.35^{* * *}$ \\
\hline \multicolumn{9}{|l|}{ Risk factors for HPV: } \\
\hline (1) Early puberty & 667 & 54.67 & 553 & 45.33 & & & & \\
\hline No & 603 & 55.63 & 481 & 44.37 & 1.00 & Reference & 1.00 & Reference \\
\hline Yes & 64 & 47.06 & 72 & 52.94 & 0.71 & $0.50 \sim 1.01$ & 0.60 & $0.39 \sim 0.90^{*}$ \\
\hline (2) Multiple sexual partners & 667 & 54.67 & 553 & 45.33 & & & & \\
\hline No & 451 & 51.13 & 431 & 48.87 & 1.00 & Reference & 1.00 & Reference \\
\hline Yes & 216 & 63.91 & 122 & 36.09 & 1.69 & $1.31 \sim 2.19^{* * *}$ & 1.78 & $1.32 \sim 2.40^{* * *}$ \\
\hline (3) Partner has multiple sexual partners & 667 & 54.67 & 553 & 45.33 & & & & \\
\hline No & 479 & 51.39 & 453 & 48.61 & 1.00 & Reference & 1.00 & Reference \\
\hline Yes & 188 & 65.28 & 100 & 34.72 & 1.78 & $1.35 \sim 2.34^{* * *}$ & 1.58 & $1.16 \sim 2.16^{* *}$ \\
\hline (4) Early initiation of sexual activity & 667 & 54.67 & 553 & 45.33 & & & & \\
\hline No & 548 & 56.09 & 429 & 43.91 & 1.00 & Reference & 1.00 & Reference \\
\hline Yes & 119 & 48.97 & 124 & 51.03 & 0.75 & $0.57 \sim 1.00^{*}$ & 0.70 & $0.51 \sim 0.96^{*}$ \\
\hline (5) Failure to use condoms & 667 & 54.67 & 553 & 45.33 & & & & \\
\hline No & 469 & 56.03 & 368 & 43.97 & 1.00 & Reference & 1.00 & Reference \\
\hline Yes & 198 & 51.70 & 185 & 48.30 & 0.84 & $0.66 \sim 1.07$ & 0.75 & $0.57 \sim 0.99^{*}$ \\
\hline (6) Who does HPV affect? & 642 & 53.95 & 548 & 46.05 & & & & \\
\hline Men & 139 & 54.94 & 114 & 45.06 & 1.00 & Reference & 1.00 & Reference \\
\hline Women & 254 & 44.25 & 320 & 55.75 & 0.65 & $0.48 \sim 0.88^{* *}$ & 0.60 & $0.43 \sim 0.83^{* *}$ \\
\hline Both & 249 & 68.60 & 114 & 31.40 & 1.79 & $1.29 \sim 2.50^{* * *}$ & 1.86 & $1.26 \sim 2.74^{* * *}$ \\
\hline (7) HPV is symptomatic & 711 & 54.07 & 604 & 45.93 & & & & \\
\hline Always symptomatic & 53 & 46.90 & 60 & 53.10 & 1.00 & Reference & 1.00 & Reference \\
\hline Sometimes & 123 & 74.55 & 42 & 25.45 & 3.32 & $1.99 \sim 5.52^{* * *}$ & 3.72 & $2.08 \sim 6.67^{* * *}$ \\
\hline Never & 26 & 26.26 & 73 & 73.74 & 0.40 & $0.23 \sim 0.72 * *$ & 0.42 & $0.22 \sim 0.79^{* *}$ \\
\hline Do not know & 509 & 54.26 & 429 & 45.74 & 1.34 & $0.91 \sim 1.99$ & 1.53 & $1.00 \sim 2.36$ \\
\hline (8) HPV is rare & 701 & 54.51 & 585 & 45.49 & & & & \\
\hline No & 615 & 62.82 & 364 & 37.18 & 1.00 & Reference & 1.00 & Reference \\
\hline Yes & 86 & 28.01 & 221 & 71.99 & 0.23 & $0.17 \sim 0.35^{* * *}$ & 0.23 & $0.17 \sim 0.31^{* * *}$ \\
\hline
\end{tabular}

Note: $* * *, * *, *$ indicating significance levels of $\leq 0.001, \leq 0.01$, and $\leq 0.05$, respectively.

$[31,32]$. But a recent study of young Asian American women [33] also reported that younger age was associated with lower likelihood of obtaining screening. With increasing age, there was an increase in odds of ever having had a Pap smear test. Our finding that younger Vietnamese women were less likely to have ever had a Pap test may be due, in part, to cultural norms that promote more conservative views about sexual relations among unmarried individuals [12]. Specifically, focus groups among young Asian Americans revealed that premarital sexual behavior was generally not widely acceptable, nor was seeking gynecological examinations prior to marriage, as that might adversely reflect sexual promiscuity [33]. As a result, younger unmarried women may be less likely to seek cervical cancer screening due to the perceived community stigma associated with sexual promiscuity. This would also be consistent with prior findings reporting the common belief that Pap testing is only for women who are married or who have had children [12]. Indeed, in the present study, we found that women who were not married or who were divorced/separated were less likely to have ever had a Pap smear test. Therefore, these findings highlight the need to focus on younger Vietnamese American women or those who are not married who may not engage in screening behaviors due to cultural barriers and beliefs.

Our findings that higher levels of education, English proficiency, employment, access to a physician, physician recommendation, and having health insurance are associated with significantly higher rates of Pap screening and are 
TABLE 5: Factors associated with receipt of prior Pap test in multivariate analyses.

\begin{tabular}{|c|c|c|c|}
\hline \multirow[t]{2}{*}{ Variable group } & \multirow[t]{2}{*}{ Associated factors } & \multicolumn{2}{|c|}{ Forward selection adjusted odds ratios } \\
\hline & & Point estimation & $95 \% \mathrm{CI}$ \\
\hline \multirow{9}{*}{ Access barriers } & Doctor recommendation for testing & $* * *$ & \\
\hline & No & 1.00 & Reference \\
\hline & Yes & $8.45^{* * *}$ & $5.02 \sim 14.23$ \\
\hline & Current health insurance & $* * *$ & \\
\hline & No & 1.00 & Reference \\
\hline & Yes & $3.04^{* * *}$ & $2.25 \sim 4.11$ \\
\hline & Difficulty getting time off to see doctor & $* * *$ & \\
\hline & No & 1.00 & Reference \\
\hline & Yes & $0.51^{* * *}$ & $0.38 \sim 0.69$ \\
\hline \multirow{23}{*}{ Knowledge, attitude, and beliefs } & Pap test can detect cancer early and prolong life & $* * *$ & \\
\hline & Yes & 1.00 & Reference \\
\hline & Do not know & $0.53^{*}$ & $0.32 \sim 0.87$ \\
\hline & No & $0.24^{* * *}$ & $0.12 \sim 0.48$ \\
\hline & Ever heard of HPV & $* * *$ & \\
\hline & No & 1.00 & Reference \\
\hline & Yes & $2.37 * * *$ & $1.56 \sim 3.59$ \\
\hline & Can have cancer but no symptoms & $* * *$ & \\
\hline & Yes & 1.00 & Reference \\
\hline & Do not know & $0.68^{*}$ & $0.48 \sim 0.97$ \\
\hline & No & 1.47 & $0.98 \sim 2.21$ \\
\hline & See doctor only when sick & $* *$ & \\
\hline & Yes & 1.00 & Reference \\
\hline & Do not know & 1.21 & $0.78 \sim 1.89$ \\
\hline & No & $1.85^{* *}$ & $1.27 \sim 2.70$ \\
\hline & God or higher power controls cancer & $*$ & \\
\hline & Yes & 1.00 & Reference \\
\hline & Do not know & $0.70^{*}$ & $0.50 \sim 0.98$ \\
\hline & No & $0.55^{* *}$ & $0.35 \sim 0.85$ \\
\hline & Cancer can be cured if detected early & * & \\
\hline & Yes & 1.00 & Reference \\
\hline & Do not know & 0.75 & $0.47 \sim 1.21$ \\
\hline & No & $0.34^{* *}$ & $0.16 \sim 0.74$ \\
\hline
\end{tabular}

Note: All demographic variables significant in the univariate logistic regression model (Table 1) were included in the multivariate model.

$* * *, * *, *$ indicating significance levels of $\leq 0.001, \leq 0.01$, and $\leq 0.05$, respectively.

corroborated by other studies $[31,34,35]$. For example, higher educational attainment was associated with greater likelihood of screening, whereas being unemployed was associated with lower likelihood of screening. These associations may be attributed, in part, to differences in knowledge and access to care. Women with higher educational levels may have greater knowledge and understanding regarding the need for cancer screening tests [36, 37]. In addition, as education level is frequently associated with income, women with higher education or who have employment may have greater financial resources with which to obtain preventive care. Women who could not speak English and who were foreign-born were less likely to have ever had a Pap smear test. This is consistent with prior studies that have reported difficulties with English as a significant obstacle to screening for this population $[38,39]$. Unfortunately, language issues continue to serve as a barrier to screening as multilingual screening services are extremely limited or unavailable for most Vietnamese American women.

Access barriers continue to remain a challenge in this population [34]. Vietnamese women who do not have a regular healthcare provider were less likely to report ever having had a Pap test, which is consistent with numerous prior studies $[16,28,31]$. Having a physician that one sees for routine healthcare over time is likely to lead to better quality of care and offers more opportunities for discussing prevention and screening options than if one only visits a healthcare provider when one is ill. Further, receiving a physician recommendation for screening was the strongest factor associated with screening, similar to prior published findings $[30,31,35]$. These findings highlight the importance of having access to a regular healthcare provider who can 
recommend the appropriate screening tests and remind women to participate in preventive care. Although programs are available that provide low-cost or free cancer screening services for women without health insurance or a healthcare provider, Vietnamese American women are often unaware of these services, not eligible for these programs, or face considerable barriers to accessing such programs. As a result, intervention programs to reduce cervical cancer disparities need to identify and target the subgroups of women who are not able to access or receive these cancer screening programs.

Our study identified 11 factors in the knowledge, attitudes, and beliefs domain that were associated with Vietnamese women's cervical cancer screening behavior. Specifically, a lack of knowledge was associated with poor participation in cervical cancer screening. Other studies have reported that Vietnamese American women may hold various misconceptions about cervical cancer and cervical cancer screening [29, 35]; as a result, these beliefs may prevent women from obtaining the necessary screening. However, women who correctly reported that a Pap smear test can detect cervical cancer early were twice as likely to have had a Pap test compared to women who did not hold this belief. Similarly, women who had heard about human papillomavirus (HPV) were more likely to have had a Pap test.

It should be noted that HPV-specific knowledge was relatively low in this population. Indeed, nearly half of the study participants did not know that HPV can cause cervical cancer or that it was sexually transmitted. In addition, many women could not identify risk factors for HPV infection and over half of the women thought that HPV infection is rare. Further, these findings likely overestimate HPV-related knowledge given that between $9 \%-18 \%$ of respondents left these items blank. However, women who did have knowledge about HPV and its risk factors were more likely to have undergone screening.

Potential limitations of the study include the crosssectional study design and using self-report to categorize prior screening behavior. In addition, our findings may not be generalizable to Vietnamese residents who are not closely engaged with their communities, and nonparticipants may have different patterns of cancer screening behaviors from the study respondents. However, the findings from the present study offer one of the largest assessments of cervical cancer screening among Vietnamese American women residing in the eastern USA and provide insights regarding identified significant factors for promoting cervical cancer screening in this population.

In summary, this study adds to the literature on cancer health disparities among Vietnamese American women and sheds light on the special health needs of this relatively recent immigrant population. Vietnamese women have the highest incidence rates of cervical cancer in the USA, and numerous factors serve as barriers to their participation in Pap test screening programs. These data help us identify the subgroup of Vietnamese American women who may be underutilizing screening services, as well as significant factors that we can address to enhance screening rates. Intervention programs need to address lack of knowledge and misconceptions regarding preventive care, language difficulties and access issues. Culturally tailored and linguistically appropriate educational materials and navigation assistance to overcome access barriers have been found to be effective in increasing screening rates in other studies [40]. Indeed, although interventions that target women's health beliefs can increase knowledge, the effectiveness of such interventions is likely to be attenuated if access barriers are not adequately addressed. Access barriers, including the cost of screening, lack of insurance, and language difficulties, pose formidable challenges to this population. Community partnerships may be helpful for reducing some access barriers by providing essential infrastructure and resources to facilitate the broad implementation of health promotion programs. In addition, overcoming access barriers may necessitate setting aside additional resources to help underserved communities obtain the recommended healthcare services and/or require changing current healthcare program guidelines, such as expanding the eligibility criteria for stateand/or federally funded programs that provide low-cost cancer screening and prevention services for underserved women.

\section{Conflict of Interests}

The Authors declare that there are no conflict of interests.

\section{Acknowledgments}

This research was partially supported by National Institutes of Health, National Cancer Institute Grants R01 CA 111570 (G. X. Ma, Ph.D., Principal Investigator) and U54 CA153513 Asian Community Cancer Health Disparities Center (PI: Dr. G. Ma). Dr. S. Ge was supported by Grant no. T32 CA09168 from the National Institutes of Health. The contents of this paper are solely the responsibility of the authors and do not necessarily represent the official views of the NCI or NIH. The authors wish to thank Asian Community Health Coalition and its member organizations for collaboration.

\section{References}

[1] Pew Research Center, Pew Social \& Demographic Trends, Pew Research Center, 2012.

[2] U.S. Census Bureau, "2010 American Community Survey," 2010, http://factfinder2.census.gov/faces/tableservices/jsf/pages/productview.xhtml?pid=ACS_10_1YR_B02006\&prodType $=$ table.

[3] J. S. Barnes, Census 2000 Brief, DIANE Publishing, 2000.

[4] A. D. Schmidley, Profile of the Foreign-Born Population in the United States, 2000, U.S. Census Bureau, U.S. Department of Commerce, Economics and Statistics Administration, 2001.

[5] B. A. Miller, K. C. Chu, B. F. Hankey, and L. A. G. Ries, "Cancer incidence and mortality patterns among specific Asian and Pacific Islander populations in the U.S.," Cancer Causes and Control, vol. 19, no. 3, pp. 227-256, 2008.

[6] M. Kagawa-Singer and N. Pourat, "Asian American and Pacific Islander breast and cervical carcinoma screening rates and healthy people 2000 objectives," Cancer, vol. 89, no. 3, pp. 696705. 
[7] S. L. Parker, T. Tong, S. Bolden, and P. A. Wingo, "Cancer statistics, 1997," CA: A Cancer Journal for Clinicians, vol. 47, no. 1, pp. 5-27, 1997.

[8] M. McCracken, M. Olsen, M. S. Chen Jr. et al., "Cancer incidence, mortality, and associated risk factors among Asian Americans of Chinese, Filipino, Vietnamese, Korean, and Japanese ethnicities," CA: Cancer Journal for Clinicians, vol. 57, no. 4, pp. 190-205, 2007.

[9] S. S. Coughlin and R. J. Uhler, "Breast and cervical cancer screening practices among Asian and Pacific Islander women in the United States, 1994-1997," Cancer Epidemiology Biomarkers and Prevention, vol. 9, no. 6, pp. 597-603, 2000.

[10] G. X. Ma, S. E. Shive, M. Q. Wang, and Y. Tan, "Cancer screening behaviors and barriers in Asian Americans," American Journal of Health Behavior, vol. 33, no. 6, pp. 650-660, 2009.

[11] H. H. Do, V. M. Taylor, N. Burke, Y. Yasui, S. M. Schwartz, and J. C. Jackson, "Knowledge about cervical cancer risk factors, traditional health beliefs, and pap testing among vietnamese american women," Journal of Immigrant and Minority Health, vol. 9, no. 2, pp. 109-114, 2007.

[12] B. J. Gor, J. A. Chilton, P. T. Camingue, and R. A. Hajek, "Young Asian Americans' knowledge and perceptions of cervical cancer and the human papillomavirus," Journal of Immigrant and Minority Health, vol. 13, no. 1, pp. 81-86, 2011.

[13] G. X. Ma, J. I. Toubbeh, M. Q. Wang, S. E. Shive, L. Cooper, and A. Pham, "Factors associated with cervical cancer screening compliance and noncompliance among Chinese, Korean, Vietnamese, and Cambodian women," Journal of the National Medical Association, vol. 101, no. 6, pp. 541-551, 2009.

[14] N. A. Ponce, N. Chawla, S. H. Babey et al., "Is there a language divide in pap test use?" Medical Care, vol. 44, no. 11, pp. 998 1004, 2006.

[15] H. S. Juon, C. Seung-Lee, and A. C. Klassen, "Predictors of regular pap smears among Korean-American women," Preventive Medicine, vol. 37, no. 6, pp. 585-592, 2003.

[16] V. M. Taylor, S. M. Schwartz, Y. Yasui et al., "Pap testing among vietnamese women: health care system and physician factors," Journal of Community Health, vol. 29, no. 6, pp. 437-450, 2004.

[17] K. Baicker, A. Chandra, and J. S. Skinner, "Geographic variation in health care and the problem of measuring racial disparities," Perspectives in Biology and Medicine, vol. 48, no. 1, supplement, pp. S42-S53, 2005.

[18] T. M. Becker, D. K. Espey, H. W. Lawson, M. Saraiya, M. A. Jim, and A. G. Waxman, "Regional differences in cervical cancer incidence among American Indians and Alaska Natives, 19992004," Cancer, vol. 113, no. 5, pp. 1234-1243, 2008.

[19] C. Y. Fang, G. X. Ma, Y. Tan, and N. Chi, "A multifaceted intervention to increase cervical cancer screening among underserved Korean women," Cancer Epidemiology Biomarkers and Prevention, vol. 16, no. 6, pp. 1298-1302, 2007.

[20] G. X. Ma, S. E. Shive, Y. Tan, and R. M. Feeley, "The impact of acculturation on smoking in Asian American homes," Journal of Health Care for the Poor and Underserved, vol. 15, no. 2, pp. 267-280, 2004.

[21] G. X. Ma, Y. Tan, J. I. Toubbeh, X. Su, S. E. Shive, and Y. Lan, "Acculturation and smoking behavior in Asian-American populations," Health Education Research, vol. 19, no. 6, pp. 615-625, 2004.

[22] G. Ma, W. Gao, C. Fang et al., "Health Belief Model and the impact on cervical cancer screening among Vietnamese Americans," under review.
[23] J. Dang, J. Lee, J. H. Tran et al., "The role of medical interpretation on breast and cervical cancer screening among Asian American and Pacific Islander women," Journal of Cancer Education, vol. 25, no. 2, pp. 253-262, 2010.

[24] J. Mock, S. J. McPhee, T. Nguyen et al., "Effective lay health worker outreach and media-based education for promoting cervical cancer screening among Vietnamese American women," American Journal of Public Health, vol. 97, no. 9, pp. 1693-1700, 2007.

[25] J. M. Moskowitz, G. Kazinets, J. M. Wong, and I. B. Tager, "“Health is strength": a community health education program to improve breast and cervical cancer screening among Korean American Women in Alameda County, California," Cancer Detection and Prevention, vol. 31, no. 2, pp. 173-183, 2007.

[26] V. M. Taylor, Y. Yasui, T. T. Nguyen et al., "Pap smear receipt among Vietnamese immigrants: the importance of health care factors," Ethnicity and Health, vol. 14, no. 6, pp. 575-589, 2009.

[27] C. R. Gresenz, J. Rogowski, and J. J. Escarce, "Community demographics and access to health care among US hispanics," Health Services Research, vol. 44, no. 5, pp. 1542-1562, 2009.

[28] S. J. McPhee, J. A. Bird, T. Davis, N. T. Ha, C. N. H. Jenkins, and B. Le, "Barriers to breast and cervical cancer screening among Vietnamese-American women," American Journal of Preventive Medicine, vol. 13, no. 3, pp. 205-213, 1997.

[29] S. J. McPhee, S. Stewart, K. C. Brock, J. A. Bird, C. N. H. Jenkins, and G. Q. Pham, "Factors associated with breast and cervical cancer screening practices among Vietnamese American women," Cancer Detection and Prevention, vol. 21, no. 6, pp. 510-521, 1997.

[30] T. T. Nguyen, S. J. McPhee, T. Nguyen, T. Lam, and J. Mock, "Predictors of cervical pap smear screening awareness, intention, and receipt among Vietnamese-American women," American Journal of Preventive Medicine, vol. 23, no. 3, pp. 207-214, 2002.

[31] I. K. Ho and K. T. Dinh, "Cervical cancer screening among Southeast Asian American women," Journal of Immigrant and Minority Health, vol. 13, no. 1, pp. 49-60, 2011.

[32] V. M. Taylor, T. T. Nguyen, J. C. Jackson, and S. J. McPhee, "Cervical cancer control research in Vietnamese American communities," Cancer Epidemiology Biomarkers and Prevention, vol. 17, no. 11, pp. 2924-2930, 2008.

[33] G. J. Yoo, M. N. Le, S. Vong, R. Lagman, and A. G. Lam, "Cervical cancer screening: attitudes and behaviors of young Asian American women," Journal of Cancer Education, vol. 26, no. 4, pp. 740-746, 2011.

[34] C. Y. Fang, G. X. Ma, and Y. Tan, "Overcoming barriers to cervical cancer screening among Asian American women," North American Journal of Medicine and Science, vol. 4, no. 2, pp. 77-83, 2011.

[35] V. Ho, J. M. Yamal, E. N. Atkinson, K. Basen-Engquist, G. Tortolero-Luna, and M. Follen, "Predictors of breast and cervical screening in Vietnamese women in Harris County, Houston, Texas," Cancer Nursing, vol. 28, no. 2, pp. 119-131, 2005.

[36] K. D. Beydag, "Knowledge and applications of the midwives and nurses at an educational hospital on the early diagnosis of cervix cancer," Asian Pacific Journal of Cancer Prevention, vol. 12, no. 2, pp. 481-485, 2011.

[37] C. Xu, W. Zhang, M. Wu, and S. Zhang, "Knowledge of cervical cancer among 25-54-year-old women in Beijing," Journal of Cancer Education, vol. 26, no. 3, pp. 555-559, 2011.

[38] S. P. Tanjasiri, J. H. Tran, M. Kagawa-Singer et al., "Exploring access to cancer control services for Asian-American and 
Pacific Islander communities in Southern California," Ethnicity and Disease, vol. 14, no. 3, supplement 1, pp. S14-S19, 2004.

[39] J. K. Yi, "Acculturation and pap smear screening practices among college-aged Vietnamese women in the United States," Cancer Nursing, vol. 21, no. 5, pp. 335-341, 1998.

[40] T. U. N. Nguyen and M. Kagawa-Singer, "Overcoming barriers to cancer care through health navigation programs," Seminars in Oncology Nursing, vol. 24, no. 4, pp. 270-278, 2008. 


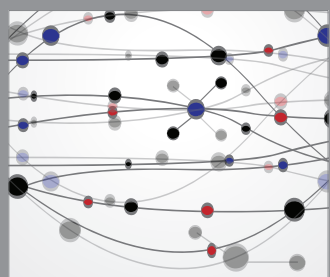

The Scientific World Journal
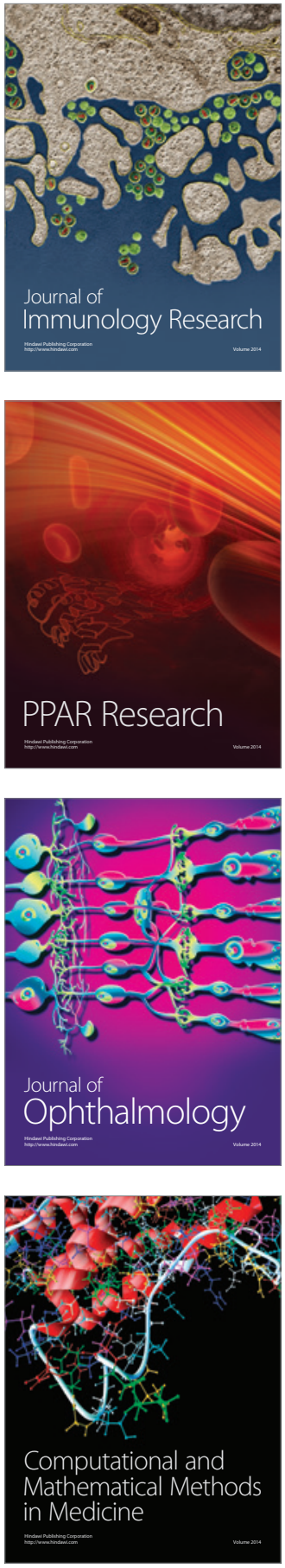

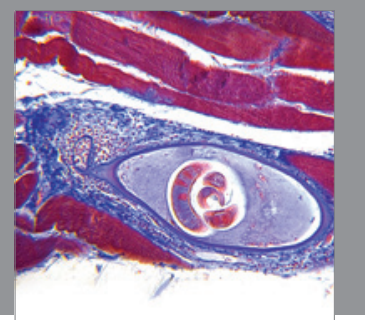

Gastroenterology

Research and Practice
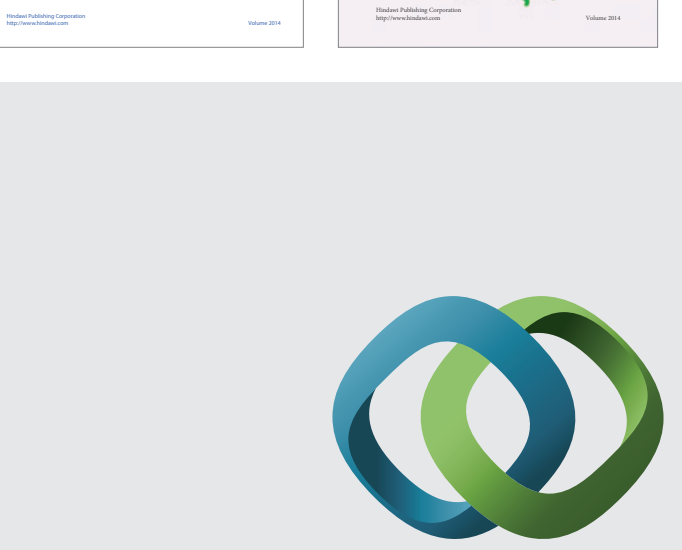

\section{Hindawi}

Submit your manuscripts at

http://www.hindawi.com
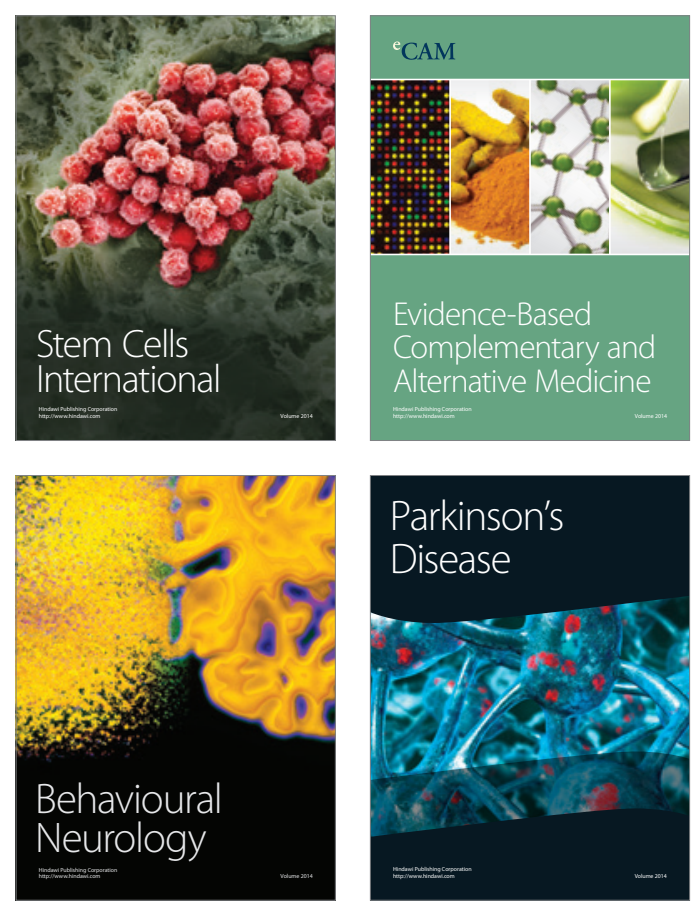

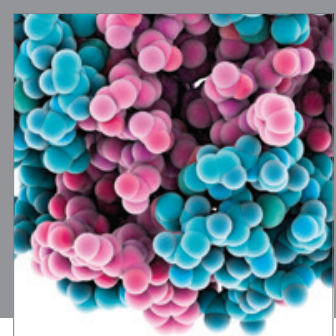

Journal of
Diabetes Research

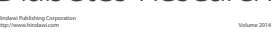

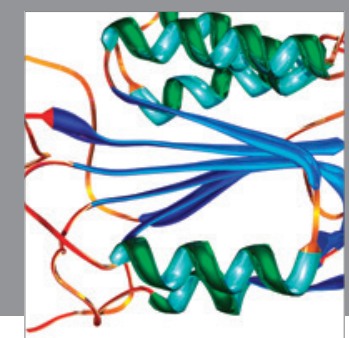

Disease Markers
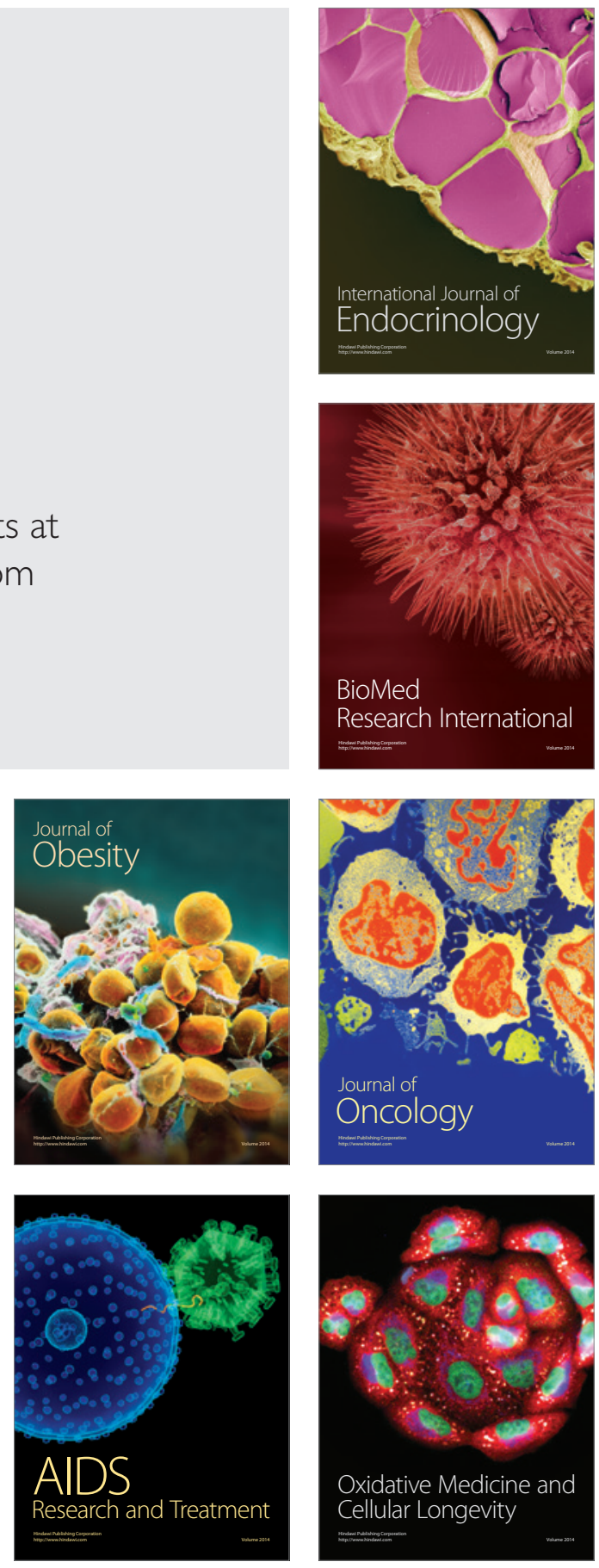